\title{
The Stokes' Theorem
}

\section{Ghiyasuddin Ghawsi}

\section{Department of Mathematics, Faculty of Education, University of Kunduz, Afghanistan}

gulistankhairandish@gmail.com

Abstract. Stokes theorem for the first presented in 1854 as a research question in Cambridge University of England by George Gabriel Stokes Irish mathematician (1819-1903). Stokes theorem is the generalized form of Green's theorem, since Green's theorem connects double integral of plane region D to curve line integral which bounded this surface as below: $\mathrm{s}$ $\int_{C} F \cdot d r=\iint_{D}\left(\frac{\partial Q}{\partial x}-\frac{\partial P}{\partial y}\right) d x d y \quad \vec{F}=P \vec{i}+Q \vec{j} \quad$ Stokes theorem relates plane integral on surface $S$ to curve line integral around the boundary curve $S$ which is spatial curve. $\int_{C} F \cdot d r=\iint_{S} C u r l F \cdot n d x=\iint_{S} \nabla x F \cdot n d x$ The physical interpretation of the Stokes' theorem is that if the vector region F over the region $\Omega$ is from differential space and $x_{0} \in \Omega$, suppose that $\mathrm{S}$ is a surface in $\Omega$ which contains the $x_{0}$ and $\mathrm{C}$ is the edge of $\mathrm{S}$ and has a standard direction. The average rotation of the vector field $\vec{F}$ in the direction $\mathrm{C}$ is equal to $\int_{C} F \cdot d r$ over the area of the surface S. CurlF $\left.\right|_{x_{0}}=\operatorname{Lim}_{r \rightarrow 0} \frac{\int C \cdot d r}{\iint d \sigma}$ In other words, the rotation of a vector around the boundary of a surface is equal to the curl flex of the vector field to the entire surface. Stokes' theorem result and applications are: It is the generalized form of Green's theorem Karel's physical concept is explained by it Calculates curve line integrals in space Proves Maxwell's laws If $\Sigma_{1}$ and $\Sigma_{2}$ be two directional surfaces bounded to curve unit $\mathrm{C}$ which both express same direction to C. $n_{1}$ and $n_{2}$ are perpendicular vectors units of $\Sigma_{1}$ and $\Sigma_{2}$. By application of Stokes' theorem we can result that for each field vector $F$ which its component function connected to the surfaces $\Sigma_{1}$ and $\Sigma_{2}$ partial derivatives. $\iint_{S}(C u r l F) n_{1} d s=\iint(C u r l F) n_{2} d s=\int_{C} F \cdot d r$ In cases where integration on a surface is difficult, maybe it is easy on other one.

Keywords. Stokes' theorem, plane integral, curve line integral, Green's theorem, vector field flax, boundary curve, directional surface, operator and tangential component 


\section{Introduction}

Stokes' theorem is the generalized form of Green's theorem. From the historical point, Green's theorem released in 1828. Stokes theorem presented in 1854 as a research question by George Gabriel Stokes in Cambridge university of England. Green's theorem connects double integral on surface region $\mathrm{D}$ to curve line integral which bounded this plane.

$$
\iint_{D}\left(\frac{\partial Q}{\partial x}-\frac{\partial P}{\partial y}\right) d x d y=\int_{C} F \cdot d r
$$

The vector function $\mathrm{F}$ is in surface $\vec{F}=P \vec{i}+Q \vec{j}$. The functions $\mathrm{P}$ and $\mathrm{Q}$, vector function component $\vec{F}$ are similarly on $\mathrm{x}$ and y axis. Stokes theorem connects plane integral on surface to spatial curve line integral which is:

$$
\iint_{S} C u r l F \cdot n d s=\iint_{S} \nabla x F \cdot n d s=\oint_{C} F \cdot d r
$$

Green's theorem, divergence theorem (Gauss theorem, Ostrrogradsky's theorem) and Stokes theorem are three important theorems of vector analysis which apply in electrical engineering, magnetic and fluid mechanic. Divergence theorem released in 1839 and Stokes theorem in 1854. This article describes the concept and definition of the theorem, its history, theorem proof, its physical explanation, the theorem application and five problem solutions. It is said that William Thomas known to Lord Kelvin English physicist sent the theorem to Stokes in 1850 .

Stokes theorem facilitates the solution of curve line integral in space, it explains curl concept and also proves Maxwell laws (English physicist).

\subsection{Research and Mythology}

Library method was used for writing this article, this article is first studied on books which are in reference list. Then the important point of the theorem compiled, edit and completed and it was written based on article writing principle.

\subsection{Historical background}

Stokes theorem is named after the Irish mathematician and physician (1819-1903). Stokes was lecturer at Cambridge university of England he done research about fluid flow in mechanic physics and the other about light. What we called Stokes theorem in fact it is discovered by William Thomas Irish physician (1824-1907) known to Lord Kelvin and he sent this theorem as a letter to Stokes in 1850 [15].

Stokes theorem first presented as research question in Cambridge university, after 1870 found its general application. The most important application this is theorem is Maxwell's laws proof physics [4]. 
Stokes theorem is the generalized form of Green's theorem. Since Green's theorem connects double integral on surface region D to curve line integral which bounded this plane as:

$$
\begin{aligned}
\int_{C} F d r=\iint_{D}\left(\frac{\partial Q}{\partial x}-\frac{\partial P}{\partial y}\right) d x d y \\
\text { Whereas } \vec{F}=P \vec{i}+Q \vec{j}[8] .
\end{aligned}
$$

Stokes theorem relates plane integral on surface $S$ to linear integral around boundary curve $\mathrm{S}$ which is spatial curve. If the spatial curve is moved in a counter-clockwise direction, the $\mathrm{S}$ surface always is on left and this is the positive direction of the curve $\mathrm{C}$. if $\mathrm{C}$ be bounded curve (not plane) which bounded part of S surface and S includes normal unit vector at it each point (except in curves or bounded points) and the curve $\mathrm{C}$ contains continuous tangent variable (except bounded points), then for each field vector of continuous derivable:

And the unit vector $\vec{n}$ of conductive cosine is equal to:

$$
n=\operatorname{Cos} \alpha i+\operatorname{Cos} \beta j+\operatorname{Cos} \gamma k
$$

Since $\operatorname{CurlF}=\nabla \times F$. We write the $C u r l F$ value and the unit vector $\vec{n}$ to relation (1):

$$
\begin{aligned}
& \oint_{C} F \cdot d r=\iint_{S}\left[\left\{\left(\frac{\partial R}{\partial y}-\frac{\partial Q}{\partial z}\right) i-\left(\frac{\partial R}{\partial x}-\frac{\partial P}{\partial z}\right) j+\left(\frac{\partial Q}{\partial x}-\frac{\partial P}{\partial y}\right) k\right\}\{\operatorname{Cos} \alpha i+\operatorname{Cos} \beta j+\operatorname{Cos} \gamma k\}\right] d s \\
& =\iint\left[\left(\frac{\partial R}{\partial y}-\frac{\partial Q}{\partial z}\right) \operatorname{Cos} \alpha-\left(\frac{\partial R}{\partial x}-\frac{\partial P}{\partial z}\right) \operatorname{Cos} \beta+\left(\frac{\partial Q}{\partial x}-\frac{\partial P}{\partial y}\right) \operatorname{Cos} \gamma\right] d s
\end{aligned}
$$

And the Stokes theorem in terms of the del symbol

$$
\oint_{C} F \cdot d r=\iint_{S}(\nabla \times F) \cdot n d s
$$

Circulation integral

In integral, the curve region is C. $r, \oint_{C} F \cdot d r$

Note: if $\mathrm{C}$ be curve in $x y$ plane and its direction be counter-clockwise and the region $\mathrm{r}$ bounded to $\mathrm{C}$, in this case $d s=d x d y$ and $\vec{F}=P \vec{i}+Q \vec{j}$.

$$
\begin{aligned}
(\text { CurlF }) \cdot n & =(\nabla \times F) \cdot n=\left(\frac{\partial}{\partial x} i+\frac{\partial}{\partial y} j\right) \times(P i+Q j) \cdot n \\
n & =k \text { since the direction of vector } \vec{n} \text { is upward, hence }
\end{aligned}
$$




$$
\begin{aligned}
& (\nabla \times F) \cdot n=\left(\frac{\partial Q}{\partial x} k-\frac{\partial P}{\partial y} k\right) \cdot k=\left(\frac{\partial Q}{\partial x}-\frac{\partial P}{\partial y}\right) \cdot k \cdot k \\
& \Rightarrow(\nabla \times F) \cdot n=\frac{\partial Q}{\partial x}-\frac{\partial P}{\partial y}
\end{aligned}
$$

In this case (Parsa, Qubali, Salehi, \& Wahidi, 2010)

$$
\oint_{C} F \cdot d r=\iint_{S}(\nabla \times f) \cdot n d s=\iint_{S}\left(\frac{\partial Q}{\partial x}-\frac{\partial P}{\partial y}\right) d x d y
$$

\section{Stokes theorem proof:}

Suppose $\mathrm{S}$ is a surface whose image on three planes specifies the coordinates of a region bounded by a simple closed curve, means the surface $S$ write as equation $Z=f(x, y)$, $x=g(y, z)$ and $y=h(x, z)$ in which $g, f$ and $h$ be continuous functions and derivable. We have to denote:

$$
\iint_{S}(\nabla \times A) n d s=\iint_{S}\left[\nabla \times\left(A_{1} i+A_{2} j+A_{3} k\right)\right] n d s=\oint_{C} A \cdot d r
$$

$\mathrm{C}$ is the boundary surface of $\mathrm{S}$.

First, we calculate the multiplication of the Hamilton $(\nabla)$ operator vector into first component of vector $\mathrm{A}\left(A_{1} i\right)$

$$
\begin{aligned}
& \nabla \times A_{1} i=\left|\begin{array}{ccc}
i & j & k \\
\frac{\partial}{\partial x} & \frac{\partial}{\partial y} & \frac{\partial}{\partial z} \\
A_{1} & 0 & 0
\end{array}\right|=\frac{A_{1}}{\partial z} j-\frac{\partial A_{1}}{\partial y} k \\
& \left(\nabla \times A_{1} i\right) n d s=\left(\frac{\partial A_{1}}{\partial z} j-\frac{\partial A_{1}}{\partial y} k\right) n d s=\left(\frac{\partial A_{1}}{\partial z} n j-\frac{\partial A_{1}}{\partial y} n k\right) d s
\end{aligned}
$$

Suppose we consider the $\mathrm{S}$ surface image on $x y$ plane, in this case the equation of $\mathrm{S}$ is $Z=f(x, y)$, on the other hand we have for each point of S surface:

$$
\vec{r}=x \vec{i}+y \vec{j}+z \vec{k} \text { hence } \vec{r}=x \vec{i}+y \vec{j}+f(x, y) \vec{k}
$$

We derive from the both sides of the y equalities:

$$
\frac{\partial r}{\partial y}=0+j+\frac{\partial f}{\partial y} \cdot k \quad \frac{\partial r}{\partial y}=j+\frac{\partial f}{\partial y} k
$$

$\frac{\partial r}{\partial y}$ is tangent vector to the surface $\mathrm{S}$ and vertical to unit $\vec{n}$ vector, therefore 
$\left\{\begin{array}{l}n \cdot \frac{\partial r}{\partial y}=n j+\frac{\partial f}{\partial y} \cdot n k \\ n \cdot \frac{\partial r}{\partial y}=0\end{array}\right.$
$n j+\frac{\partial f}{\partial y} \cdot n k=0 \rightarrow n j=-\frac{\partial f}{\partial y} n k$

We let $n j$ value to relation (1):

$\left(\nabla \times A_{1} i\right) n d s=\left[\frac{\partial A_{1}}{\partial z}\left(-\frac{\partial f}{\partial y} n k\right)-\frac{\partial A_{1}}{\partial y} n k\right] d s=\left(-\frac{\partial A_{1}}{\partial z} \cdot \frac{\partial z}{\partial y} n k-\frac{\partial A_{1}}{\partial y} n k\right) d s$
$\left(\nabla \times A_{1} i\right) n d s=-\left(\frac{\partial A_{1}}{\partial y}+\frac{\partial A_{1}}{\partial z} \cdot \frac{\partial z}{\partial y}\right) n k d s \quad \ldots$.

We can write on the surface $S$ :

$A_{1}(x, y, z)=A_{1}(x, y, f(x, y))=F(x, y)$

We derive from the both sides of the y equalities:

$\frac{\partial A_{1}}{\partial y}+\frac{\partial A_{1}}{\partial z} \cdot \frac{\partial z}{\partial y}=\frac{\partial F}{\partial y} \ldots$

We let the left side value of (3) into relation (2):

$\left(\nabla \times A_{1} i\right) n d s=-\frac{\partial F}{\partial y} n k d s \quad, \quad \vec{n} \cdot \vec{k}=1$

hence

$\left(\nabla \times A_{1} i\right) n d s=-\frac{\partial F}{\partial y} d s=-\frac{\partial F}{\partial y} d x d y$

$\iint_{S}\left(\nabla \times A_{1} i\right) n d s=-\iint_{R} \frac{\partial F}{\partial y} d x d y$

$\mathrm{R}$ is the image of the surface $\mathrm{S}$ on the plane $x y$, according to the Green theorem in the plane, the integral $-\iint_{R} \frac{\partial F}{\partial y} d x d y$ can be written as $\int_{\Omega} F d x$, where $\Omega$ is the boundary of the region $\mathrm{R}$. Since each point $(x, y)$ in the boundary curve of region $\mathrm{R}$, that is, in $\Omega$, is equal to $\mathrm{F}$, several values of $A_{1}$ are at each point $(x, y, z)$ in $\mathrm{C}$, and $d x$ is the same value for the two-dimensional curves $\Omega$ and the three-dimensional curve $\mathrm{C}$, so 


$$
\begin{aligned}
\oint_{\Omega} F d x & =\oint_{C} A_{1} d x \\
& \text { Or } \iint_{S}\left[\nabla \times\left(A_{1} i\right)\right] n d s=\oint_{C} A_{1} d x \quad \ldots . I
\end{aligned}
$$

Similarly, if we do this calculation on the other two planes of the perpendicular threedimensional coordinates in plane $y z$ and plane $x z$, we get that:

$$
\begin{aligned}
& \iint_{S}\left[\nabla\left(A_{2} \vec{j}\right)\right] n d s=\oint_{C} A_{2} d y \quad \ldots . \quad I I \\
& \iint_{S}\left[\nabla\left(A_{3} \vec{k}\right)\right] n d s=\oint_{C} A_{3} d z \quad \ldots .
\end{aligned}
$$

By summing these three integrals, we get that

$$
\iint_{S}(\nabla \times A) n d s=\oint_{C} A d r
$$

Hence, this completes the proof of the Stokes theorem [6].

\subsection{Physical interpretation of the Stokes theorem}

If the vector field $\vec{F}$ on the region $\Omega$ is from differential space and is $x_{0} \in \Omega$, suppose that $S$ is a surface in $\Omega$ that contains $x_{0}$ as a regular point and $C$ is the edge of $S$ and has a standard direction. In this case, the mean rotation of the vector area $\vec{F}$ in the direction $\mathrm{C}$ is equal to the outside of part $\oint_{C} F \cdot d r$ on the area of the surface $\mathrm{S}$.

The Limit form of this value is called the rotation of $\mathrm{F}$ at $\mathrm{x}$ when $\mathrm{S}$ approximate to $x_{0}$, and is written with the symbol $\left.\operatorname{CurlF}\right|_{x_{0}}$.

$$
\text { Curl }\left.F\right|_{x_{0}}=\operatorname{Lim}_{r \rightarrow 0} \frac{\oint_{C} F \cdot d r}{\iint_{S} d \sigma}
$$

If $\mathrm{S}$ is the directional and edged surface with edge $\mathrm{C}$ and $\mathrm{C}$ is the standard direction, the vector field $\vec{F}$ is still derivative of $\mathrm{S}$ and is connected to the curve $\mathrm{C}$, the work done by the dynamic object based on $\mathrm{C}$ under the influence of the vector field $\vec{F}$ is equal to rotation scale F across the surface of $S$ [10].

The concept of the Stokes theorem without mathematical expression is that: the rotation of a vector around the boundary of a surface is equal to the curl flex of a vector field to the entire of this surface [12]. 


\subsection{Ampere's Circuital Law}

The ampere's circuital law can be obtained from $\operatorname{Curl} \vec{H}=\vec{\nabla} \times \vec{H}=j$. In the following figure, we divide the $S$ surface into small areas. If we apply the curl definition to one of the surface samples, we will have.

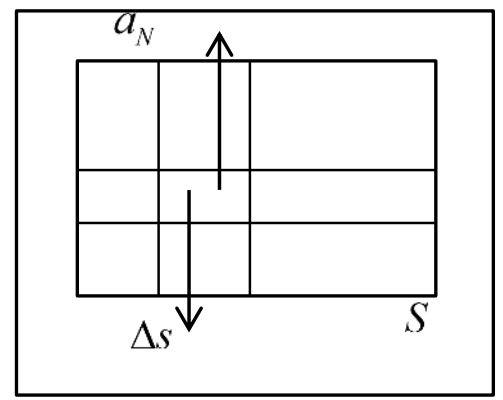

$$
\int_{C} \frac{H \cdot d L_{\Delta s}}{\Delta s}=(\nabla \times H)_{N}
$$

Figure 1

$\mathrm{H}$ is the magnitude of the magnetic field and $\vec{j}$ is the velocity vector of the current.

$\mathrm{N}$ denotes the perpendicular to the surface, which is determined by the law of the right hand. $d L_{\Delta s}$ means that the path is closed to the environment. $\mathrm{N}$ is a unit vector perpendicular to the small surface $\mathrm{x}$.

$$
\begin{aligned}
& \int_{C} \frac{H \cdot d L_{\Delta s}}{\Delta s}=(\nabla \times H) \cdot a_{N} \\
& \int_{X} H \cdot d L_{\Delta s}=(\nabla \times H) \cdot a_{N} \cdot \Delta s=(\text { CurlH }) \cdot \Delta s
\end{aligned}
$$

We also sum the linear integrals on the closed path for all $\Delta s$, resulting in the addition of omissions, because each inner wall travels in two different directions, so that

$$
\oint_{C} H \cdot d L=\iint_{S}(\nabla \times H) d s
$$

Equation (1) is a vector union that is true for any vector field and is known as the Stokes theorem [5].

\subsection{Results and applications of the Stokes theorem}

Applications of the Stokes theorem include at least three of the following.

1. The Green theorem in the plane as a special form of the Stokes theorem

Suppose $\vec{V}=V_{1} \vec{i}+V_{2} \vec{j}$ is a function of a vector that is continuously derivable in an area of plane $x y$ that contains the closed region of the simple interconnection $\mathrm{S}$ where $\mathrm{C}$ is a closed curve and smooth fragment, hence 


$$
\begin{aligned}
& (\text { CurlV })_{n}=\frac{\partial V_{2}}{\partial x}-\frac{\partial V_{1}}{\partial y} \\
& \iint_{S}(\operatorname{Curl} C)_{n} d A=\iint_{S}\left(\frac{\partial V_{2}}{\partial x}-\frac{\partial V_{1}}{\partial y}\right) d A=\int_{C} V_{1} d x V_{2} d y
\end{aligned}
$$

The last mathematical expression means that the Green theorem in the plane is a special form of the Stokes theorem [9].

\section{Curl Physical Interpretation}

If $\mathrm{F}$ is a vector field like the fluid velocity field, then the rotation $\oint_{C} F \cdot d r$ corresponding to $\mathrm{F}$ around the edge of $\mathrm{C}$ is a measure of the tendency of the fluid to rotate around or rotate around it. To better understand this, suppose that $P_{0}\left(x_{0}, y_{0}, z_{0}\right)$ is an alternative point in fluid, $C_{r}$ is a small circle with radius $\mathrm{r}$ and center $P_{0}$. We have the Stokes theorem (1). $\int_{C_{r}} F \cdot d r=\iint_{S_{r}} \operatorname{CurlF} \cdot n \cdot d s \quad \ldots \quad(1)$

Now at all points $P(x, y, z)$ inside the small circle $C_{r}$ if $\operatorname{CurlF} \approx \operatorname{CurlF}\left(P_{0}\right)$ is assumed, the relation leads to the following approximation

Curl Physical Interpretation

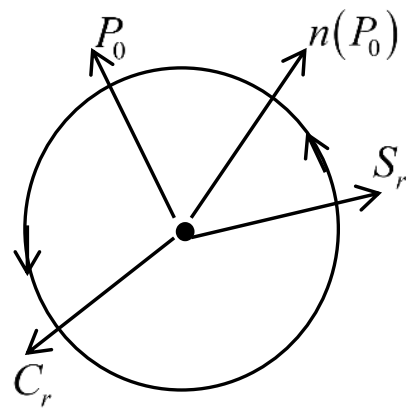

$$
\begin{aligned}
\oint_{C_{r}} F \cdot d r & \approx \iint_{S_{r}} \operatorname{CurlF}\left(P_{0}\right) \cdot n\left(P_{0}\right) d s \\
& =\operatorname{CurlF}\left(P_{0}\right) \cdot n\left(P_{0}\right) \cdot \iint_{S_{r}} d s=\operatorname{CurlF}\left(P_{0}\right) \cdot n\left(P_{0}\right) \cdot A_{r} \quad \ldots
\end{aligned}
$$

Figure 2

$A_{r}$ is the area of $\pi r^{2}$ is the circular surface of $S_{r}$, although $r \rightarrow 0$ the $F(P) \approx \operatorname{CurlF}\left(P_{0}\right)$ approximation get better, and we write equation (2) as follows.

$\operatorname{CurlF}\left(P_{0}\right)=\operatorname{Lim}_{r \rightarrow 0} \frac{1}{A_{r}} \oint_{C_{r}} F \cdot d r$

So curl $\mathrm{F}$ is the rotation of $\vec{F}$ over unit surface [15].

3. Calculation of curve line integral by Stokes theorem

Problem: Calculate the curve line integral $\int_{C} \vec{F} \cdot d \vec{r}$, where $F(x, y, z)=-y^{2} \vec{i}+x \vec{j}+z^{2} \vec{k}$ and $\mathrm{C}$ are curves consisting of the plane $y+z=2$ and the cylinder $x^{2}+y^{2}=1$. 
Solution: The following figure shows that the curve $\mathrm{C}$ is an ellipse.

We use the Stokes theorem to solve the curve line integral. $\quad y+z=2$

$$
\operatorname{Curl} \vec{F}=\left|\begin{array}{ccc}
i & j & k \\
\frac{\partial}{\partial x} & \frac{\partial}{\partial y} & \frac{\partial}{\partial z} \\
-y^{2} & x & z^{2}
\end{array}\right|=(1 \quad \text { Figure 3 }
$$

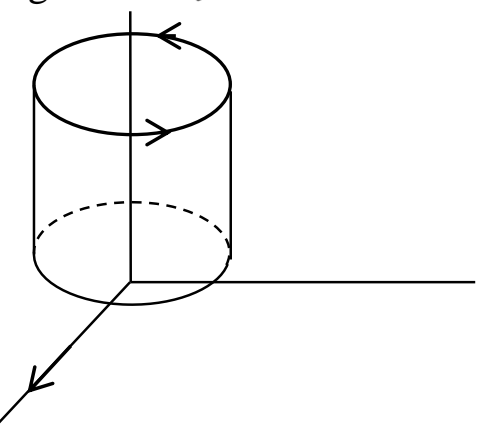

The surface $\mathrm{S}$ is the elliptical region in the plane $y+z=2$ bounded by the $\mathrm{C}$-curve. The drawing region of the $\mathrm{S}$ is above the plane $x y$ of the surface $x^{2}+y^{2} \leq 1$.

$$
\int_{C} \vec{F} \cdot d \vec{r}=\iint_{S} \operatorname{Curl} \vec{F} \cdot d \vec{s}=\iint_{S}(1+2 y) d A
$$

We solve the last integral in polar coordinates.

$$
\begin{aligned}
& y=r \operatorname{Sin} \theta \\
& d A=r d r d \theta
\end{aligned}
$$

$$
0 \leq \theta \leq 2 \pi \quad \iint_{S}(1+2 y) d A=\int_{0}^{2 \pi} \int_{0}^{1}(1+2 r \operatorname{Sin} \theta) r d r d \theta
$$

$$
=\int_{0}^{2 \pi}\left[\left.\left(\frac{r^{2}}{2}+2 \frac{r^{3}}{3} \operatorname{Sin} \theta\right)\right|_{0} ^{1}\right] d \theta=\int_{0}^{2 \pi}\left(\frac{1}{2}+\frac{2}{3} \operatorname{Sin} \theta\right) d \theta=\frac{1}{2} \cdot 2 \pi=\pi
$$

$\Rightarrow \int_{C} F \cdot d r=\pi$

$[8]$.

The concept of the Stokes theorem without mathematical expression is that the rotation of a vector around the boundary of a surface is equal to the curl flex of a vector field over this entire surface [12].

Note: If $\Sigma_{1}$ and $\Sigma_{2}$ are two directional surfaces bounded by a unit curve $\mathrm{C}$ that both induce the same direction to $\mathrm{C}$, if $n_{1}$ and $n_{2}$ are perpendicular $\Sigma_{1}$ and $\Sigma_{2}$, respectively, using the Stokes theorem, it can be concluded that for each vector field $\mathrm{F}$ whose component functions are connected to surfaces $\Sigma_{1}$ and $\Sigma_{2}$ by partial derivatives.

$$
\iint_{\Sigma_{1}}(C u r l F) n_{1} d s=\int_{C} F \cdot d r=\iint_{\Sigma_{2}}(C u r l F) n_{2} d s
$$

Therefore, in cases where integration is difficult on one surface. It may run easily over another surface [4]. 


\subsection{Surface integral properties of vector functions}

The properties of vector or surface integral flow are vector functions such as the properties of double integrals. Here are some properties.

1. General property of vector flux

If $S$ surface is $S=S_{1} \cup S_{2}$

$$
\iint_{S} \vec{F} \cdot \vec{n} d s=\iint_{S_{1}} \vec{F} \cdot \vec{n} d s+\iint_{S_{2}} \vec{F} \cdot \vec{n} d s
$$

2. If a surface is converted to the opposite surface, the vector flux notation changes.

$$
\iint_{S} \vec{F} \cdot \vec{n} d s=-\iint_{S} \vec{F} \cdot \overrightarrow{n^{\prime}} d s
$$

$\overrightarrow{n^{\prime}}$ is a normal unit whose direction is opposite to the direction of vector $\vec{n}$. The double integral does not have this property [3].

Problem 1: If the velocity of a fluid with impurity $\rho=1 \frac{\mathrm{g}}{\mathrm{cm}^{3}}$ is equal to $F(x, y, z)=5 z k$ and $\Delta$ is the surface of the sphere $x^{2}+y^{2}+z^{2}=16$, find the amount of fluid flow from the surface of this sphere?

Solution: If we name $\mathrm{D}$ the image of the sphere on the $x y$ page, the region $\mathrm{D}$ is in the circle $x^{2}+y^{2}=16$.

Since the vector field $\mathrm{F}$ is $\vec{F}=\vec{F}_{1} i+\vec{F}_{2} j+\vec{F}_{3} k$ and in the presented problem which $F=5 z k$. From the comparison of these it is resulted: $F_{3}=5 z, F_{2}=0, F_{1}=0$. The amount of

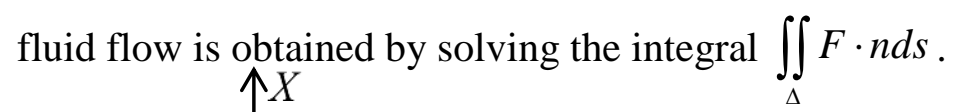

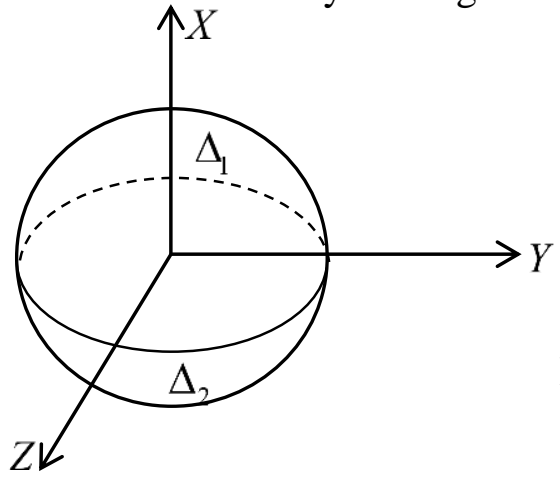

$$
\iint_{\Delta} F \cdot n d s=\iint_{\Delta_{1}} F \cdot n d s+\iint_{\Delta_{2}} F \cdot n d s
$$

Figure 4

$\Delta_{1}$ and $\Delta_{2}$ are the upper and lower hemispheres, respectively, $\overrightarrow{n_{1}}$ is a unit vector perpendicular to $\Delta_{1}$ and $\vec{n}$ is a vector unit perpendicular to $\Delta_{2}$. 
The function $Z=\sqrt{16-x^{2}-y^{2}}$, if it is $z=g(x, y)$, then $g(x, y)=\sqrt{16-x^{2}-y^{2}}$. Since in the upper hemisphere the direction of the unit vector $\vec{n}$ is upwards. Therefore, the following formula is used:

$$
\begin{aligned}
\iint_{\Delta_{1}} F \cdot d s & =\iint_{D}\left(-F_{1} \cdot g_{x}-F_{2} \cdot g_{y}+F_{3}\right) d s \\
& =\iint_{D}\left(-0 \cdot g_{x}-0 \cdot g_{y}+5 z\right) d s=5 \iint_{D} z d s=5 \iint_{D} \sqrt{16-x^{2}-y^{2}} d s
\end{aligned}
$$

We solve the $\iint_{D} \sqrt{16-x^{2}-y^{2}} d s$ integral in polar coordinates:

$$
\begin{aligned}
& x^{2}+y^{2}=r^{2} \quad, \quad d s=r d r d \theta \quad, \quad 0 \leq x^{2}+y^{2} \leq 16 \rightarrow 0 \leq r^{2} \leq 16 \\
& \rightarrow 0 \leq r \leq 4 \rightarrow 5 \iint_{D} \sqrt{16-x^{2}-y^{2}} d s=5 \int_{0}^{2 \pi} \int_{0}^{4} \sqrt{16-r^{2}} r d r d \theta
\end{aligned}
$$

Now we solve the $\int_{0}^{4} \sqrt{16-r^{2}} r d r$

suppose

$16-r^{2}=t \rightarrow-2 r d r=d t \rightarrow r d r=-\frac{1}{2} d t$

For $t=16, \quad r=0$ and for $r=4$ the $(t=0) t$ get zero, then

$$
\begin{aligned}
& \int_{0}^{r} \sqrt{16-r^{2}} r d r=\int_{16}^{0} t^{\frac{1}{2}}\left(-\frac{1}{2}\right) d t=-\frac{1}{2} \int_{0}^{16} t^{\frac{1}{2}} d t=\frac{1}{2} \int_{0}^{16} t^{\frac{1}{2}} d t=\left.\frac{1}{2} \cdot \frac{t^{\frac{3}{2}}}{\frac{3}{2}}\right|_{0} ^{16}=\frac{64}{3} \\
& \Rightarrow 5 \int_{0}^{2 \pi} \int_{0}^{4} \sqrt{16-r^{2}} r d r d \theta=5 \int_{0}^{2 \pi} \frac{64}{3} d \theta=5 \cdot \frac{64}{3} \cdot 2 \pi=\frac{640 \pi}{3} \\
& =\frac{640 \pi}{3}=\frac{\text { mass unit }}{\text { area unit } \cdot \text { Sec }}
\end{aligned}
$$

In MKS system this amount is equal to $\frac{640 \pi}{3} \frac{\mathrm{kg}}{\mathrm{m}^{2} \cdot \mathrm{Sec}}$, similarly the amount of fluid flow in the lower hemisphere is also equal to $\frac{640 \pi}{3}=\frac{\mathrm{kg}}{\mathrm{m}^{2} \cdot \operatorname{Sec}}$ and the $\iint_{D} F \cdot n d s$ integral solution is equal to:

$\iint_{\Delta} F \cdot n d s=\frac{640 \pi}{3} \frac{\mathrm{kg}}{\mathrm{m}^{2} \cdot \mathrm{Sec}}+\frac{640 \pi}{3} \frac{\mathrm{kg}}{\mathrm{m}^{2} \cdot \mathrm{Sec}}=\frac{1280 \pi}{3} \frac{\mathrm{kg}}{\mathrm{m}^{2} \cdot \mathrm{Sec}}$ 
Problem 2: Using the Stokes theorem, calculate the integral $\iint_{S} C u r l F d s$, which is $F(x, y, z)=x z i+y z j+x y k$ here and $\mathrm{S}$ is the part of sphere $x^{2}+y^{2}+z^{2}=4$ that lies inside a cylinder with base $x^{2}+y^{2}=1$ at the top of the plane $x y$ ?

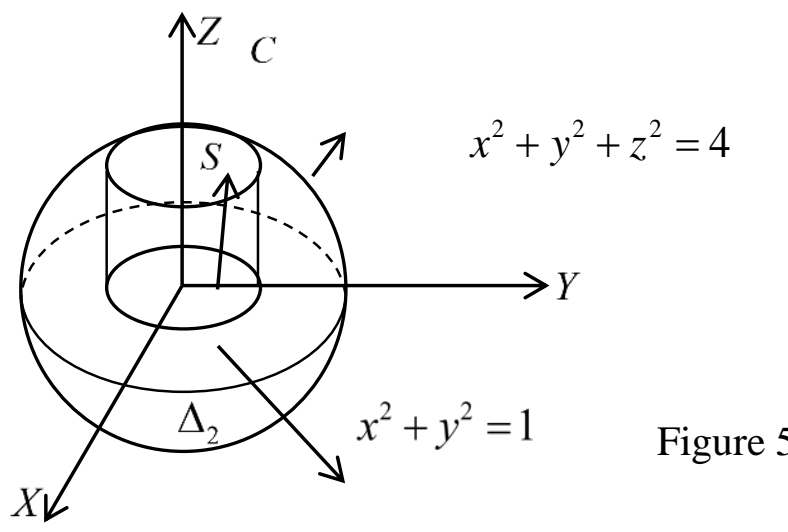

Solution: To get the space curve C, we solve the equations $x^{2}+y^{2}+z^{2}=4$ at the same time:

$$
\left\{\begin{array}{rl}
x^{2}+y^{2}+z^{2} & =4 \\
\pm x^{2} \pm y^{2} & = \pm 1
\end{array} \quad \rightarrow z=\sqrt{3}\right.
$$

It seems that $\mathrm{C}$ is a circle that is determined by $x^{2}+y^{2}=1$ and $z>0$ equations. The vector equation of the spatial curve $\mathrm{C}$ is:

$r(t)=x(t) i+y(t) j+z(t) k$

And the parametric equations of the circle $x=r \operatorname{Cos} t$ and $y=r S$ int are $r=1$ since $x^{2}+y^{2}=1=r^{2}$ hence

$$
\begin{aligned}
& r(t)=\cos t i+\sin t j+\sqrt{3} k=(\cos t, \sin t, \sqrt{t}) \\
& r^{\prime}(t)=-\sin t i+\cos t j
\end{aligned}
$$

And now we find the $F(r(t))$ value as follows:

$F(x, y, z)=x z i+y z j+x y k$

$F(r(t))=F(\cos t, \sin t, \sqrt{3})=\sqrt{3} \cos t i+\sqrt{3} \sin t j \cos t k$ 


$$
\begin{aligned}
& \iint_{C} \operatorname{curl} F \cdot d s=\int_{C} F d s=\int_{0}^{2 \pi} F(r(t)) \cdot r^{\prime}(t) d t=\int_{0}^{2 \pi}(\sqrt{3} \cos t i+\sqrt{3} \sin t j+\sin t \cos t k)(-\sin t i+\cos t j) d t \\
& \int_{0}^{2 \pi}(-\sqrt{3} \sin t \cos t+\sqrt{3} \sin t \cos t+0) d t=\int_{0}^{2 \pi} 0 \cdot d t=0 \\
& \Rightarrow \iint_{S} c u r l F d s=0
\end{aligned}
$$

Problem 3: Investigate the validity of the Stokes theorem for the vector field $F(x, y, z)=\left(e^{x}, e^{y}, e^{z}\right)$ while the surface $\mathrm{S}$ is defined by the two variable functions $Z=1-x^{2}-y^{2}$ for $Z \geq 0$ and $\mathrm{n}$ is an external normal vector?

Solution: The Stokes theorem is $\int_{C} P d x+Q d y+R d z=\iint_{S} C u r l \vec{F} \vec{n} d \vec{s}$. To denote the equality of both sides, we must calculate the curve line integral on the left and the surface integral on the right.

$$
\begin{array}{r}
\text { CurlF }=\left|\begin{array}{ccc}
i & j & k \\
\frac{\partial}{\partial x} & \frac{\partial}{\partial v} & \frac{\partial}{\partial z}
\end{array}\right|=i\left(\frac{\partial}{\partial v} e^{z}-\frac{\partial}{\partial z} e^{y}\right)-j\left(\frac{\partial}{\partial x} e^{z}-\frac{\partial}{\partial z} e^{x}\right)+k\left(\frac{\partial}{\partial x} e^{y}-\frac{\partial}{\partial v} e^{x}\right)=(0,0,0) \\
=\operatorname{Curl} F=(0,0,0)
\end{array}
$$

Then $\operatorname{CurlF}=(0,0,0)$

The $n$ vector value is $\vec{n}=\left(-\frac{\partial z}{\partial x},-\frac{\partial z}{\partial y}, 1\right)=(2 x, 2 y, 1)$

$$
\iint_{S} \operatorname{CurlF} \cdot n d s=\iint(0,0,0) \cdot(2 x, 2 y, 1) d x d y=0
$$

To calculate the curve line integral, we must specify the plane S. S is a circle with a radius of one unit and the center of origin, and in plane $x y$, which moves counter-clockwise, hence $z=0$ and $d z=0$.

$$
I=\int P d x+Q d y=\int e^{x} d x+e^{y} d y
$$

We solve the recent integral in polar coordinates $x=\operatorname{Cos} \theta, r=1$, $d x=-\operatorname{Sin} \theta d \theta, y=\operatorname{Sin} \theta$ and $0 \leq \theta \leq 2 \pi, d y=\operatorname{Cos} \theta d \theta$ 


$$
\begin{aligned}
& I=\int e^{\cos \theta}(-\sin \theta d \theta)+e^{\sin \theta}(\cos \theta d \theta)=\int d e^{\cos \theta}+d e^{\sin \theta}=\int_{0}^{2 \pi} d\left(e^{\cos \theta}+e^{\sin \theta}\right) \\
& I=\left.\left(e^{\cos \theta}+e^{\sin \theta}\right)\right|_{0} ^{2 \pi}=e^{\cos 2 \pi}+e^{\sin 2 \pi}-\left(e^{\cos 0}+e^{\sin 0}\right)=e+e^{0}-e-e^{0}=0
\end{aligned}
$$

Problem 4: a. Explain the Stokes theorem?

b. Write this theorem in the Cartesian coordinate system?

Solution: Part A: The Stokes theorem is that the curve line integral of the tangent component of vector $\vec{A}$ around a simple closed curve $\mathrm{C}$ is equal to the surface integral of the vertical curl compound $A$ above the surface $S$ bounded by the $C$ curve.

Part B: The $\vec{A}$ vector: $\vec{A}=A_{1} \vec{i}+A_{2} \vec{j}+A_{3} \vec{k}$

$\vec{n}$ unit vector is: $\vec{n}=\operatorname{Cos} \alpha \vec{i}+\operatorname{Cos} \beta \vec{j}+\operatorname{Cos} \gamma \vec{k}$

$$
\text { Curl } A=\nabla \times A=\left|\begin{array}{ccc}
i & j & k \\
\frac{\partial}{\partial x} & \frac{\partial}{\partial y} & \frac{\partial}{\partial z} \\
A_{1} & A_{2} & A_{3}
\end{array}\right|=\vec{i}\left(\frac{\partial}{\partial y} A_{3}-\frac{\partial}{\partial z} A_{2}\right)-\vec{j}\left(\frac{\partial}{\partial x} A_{3}-\frac{\partial}{\partial z} A_{1}\right)+\vec{k}\left(\frac{\partial}{\partial x} A_{2}-\frac{\partial}{\partial y} A_{1}\right)
$$

$\operatorname{Curl} \vec{A} \cdot \vec{n}=\left[\vec{i}\left(\frac{\partial}{\partial y} A_{3}-\frac{\partial}{\partial z} A_{2}\right)-\vec{j}\left(\frac{\partial}{\partial x} A_{3}-\frac{\partial}{\partial z} A_{1}\right)+\vec{k}\left(\frac{\partial}{\partial x} A_{2}-\frac{\partial}{\partial y} A_{1}\right)\right]$

$$
[\operatorname{Cos} \alpha \vec{i}+\operatorname{Cos} \beta \vec{j}+\operatorname{Cos} \gamma \vec{k}]
$$

$\operatorname{Curl} \vec{A} \cdot \vec{n}=\left(\frac{\partial}{\partial y} A_{3}-\frac{\partial}{\partial z} A_{2}\right) \operatorname{Cos} \alpha+\left(\frac{\partial}{\partial z} A_{1}-\frac{\partial}{\partial x}\right) \operatorname{Cos} \beta+\left(\frac{\partial}{\partial x} A_{2}-\frac{\partial}{\partial y} A_{1}\right) \operatorname{Cos} \gamma$

On the other hand the curve line integral $\int_{C} \vec{A} \cdot d \vec{r}$ is equal to

$$
\begin{aligned}
& \int_{C} \vec{A} \cdot d \vec{r}=\int_{C}\left(A_{1} \vec{i}+A_{2} \vec{j}+A_{3} \vec{k}\right) \cdot(d x \vec{i}+d y \vec{j}+d z \vec{k})=\int_{C} A_{1} d x+A_{2} d y+A_{3} d z \\
& \int_{C} \vec{A} \cdot d \vec{r}=\iint_{S} \operatorname{Curl} \vec{A} \cdot \vec{n} \cdot d s
\end{aligned}
$$

or

$$
\int_{C} A_{1} d x+A_{2} d y+A_{3} d z=\iint\left(\frac{\partial}{\partial y} A_{3}-\frac{\partial}{\partial z} A_{2}\right) \cos \alpha+\left(\frac{\partial}{\partial z} A_{1}-\frac{\partial}{\partial x} A_{3}\right) \cos \beta+\left(\frac{\partial}{\partial x} A_{2}-\frac{\partial}{\partial y} A_{1}\right) \cos \gamma
$$




\section{Findings}

Historical records state that this was not the case with Stokes at all; it was sent to Stokes by William Thomas, the English physicist Lord Calvin in 1850, and by George Gabriel Stokes, an Irish mathematician and physicist (1903-1819). The case was raised in 1854 as a research question at the University of Cambridge in England, and has been referred to as the Stokes case ever since.

The Green theorem published in 1828, the divergence theorem (Gauss theorem, Strogerdski's theorem) published in 1839, and the Stokes theorem are important theorems of vector analysis used in fluid mechanics and electrical and magnetic engineering.

The complex problems of spatial curve line integrals are easily solved by this theorem. Maxwell's laws of the English physicist (1879-1813) as expressed by Divergence and Curl of electric and magnetic fields: $\nabla \times E=-\frac{\partial H}{\partial t}, \nabla \vec{H}=\operatorname{div} \vec{H}=0, \operatorname{div} \vec{E}=\nabla \vec{E}=\frac{\rho}{\varepsilon} \quad$ And $\nabla \times \vec{H}=\operatorname{Curl} \vec{H}=\delta+\frac{1}{C^{2}} \frac{\partial E}{\partial t}$ are proved by the Stokes theorem, as well as the physical interpretation of curl by this theorem.

\section{Discussion}

Stokes' theorem is one of the most important theorems in vector analysis, which is widely used in magnetic physics, electricity, and fluid dynamics. This theorem transforms the complex problems of spatial curve line integrals into the surface integral of the same surface that surrounds the spatial curve, thus facilitating their solution.

It is the generalized form of Green theorem. Curl's physical interpretation is done by it. Historically published after Green and Divergence theorems (Gauss's theorem, Strogradsky theorem).

This theorem is present in most textbooks of vector analysis and higher mathematics due to its importance and applications. In writing this article, I used 15 fifteen titles of books related to higher mathematics.

\section{Conclusion}

Stokes' theorem is an important theorem for vector analysis that's a major issue in most high school math textbooks because of its importance and applications in fluid dynamics and electrical and magnetic engineering. History of Mathematics states that the theorem was invented by the English physicist William Thomson and sent in a letter to George Gabriel Stokes in 1850. The generalized form is the Green theorem.

This theorem has been historically developed after the Green and Divergence theorems. Knowing this theorem and applying it in physics will help the esteemed reader of advanced 
mathematics to teach advanced engineering mathematics, fluid dynamics, and teaching problems related to vector fields.

\section{References:}

[1]. Far, A. S. (2006). General mathematics (2) (9th ed.). Tehran: Dalang.

[2]. Ghoori, M. A. (2006). Higher mathematics. Kabul: Numani.

[3]. Hamta, N. M. (2009). Higher mathematics (1st ed.). Kabul: Saeed.

[4]. Hassani, A. R., \& Neya, F. I. (2008). General mathematics (2nd ed.). Tehran: Afiz.

[5]. Hayt, W. (2001). Engineering magnet (7th ed.). (E. Jalayeri, \& B. Qahraman, Trans.) Mashad: Numa.

[6]. Kazimi, M. H. (2000). General mathematics applications. Tehran: Nai.

[7]. Khalili, A. W. (2016). Frequent integral (Vol. 1st). Kabul: Saeed.

[8]. Kohee, K. D. (2014). Vector analysis (2nd ed.). Kabul: Qurtaba.

[9]. Kreyszig, E. (1997). Advanced engineering mathematics (1st ed., Vol. 1st). (A. S. Far, \& H. Farman, Trans.) Tehran: Danesh Gahee.

[10]. Najafikha, M. (2006). General mathematics (1-2) (4th ed.). Tehran: Sahel Andisha.

[11]. Parsa, I., Qubali, Z., Salehi, A., \& Wahidi, A. R. (2010). General mathematics (2) (4th ed.). Karj: Sar Afraz.

[12]. Piskunov, N. (1996). Calculus (Vol. 2nd). (3rd, Ed., \& A. S. Far, Trans.) Tehran: Elmu Sanhat university.

[13]. Salili, S. (2007). Calculus and multivariable function integral (4th ed.). Tehran: Payame Noor university.

[14]. Stewart, J. (2010). Calculus (Vol. 2nd). (A. Amidi, Trans.) Tehran: Fatami.

[15]. Zill, D. G., \& Cullen, M. R. (2000). advanced engineering mathematics (1st ed., Vol. 1st). (A. S. Far, \& H. Waisi, Trans.) Tehran: Khane Danish Gahi Emam Hussain. 